www.jmscr.igmpublication.org

Impact Factor 5.84

Index Copernicus Value: 71.58

ISSN (e)-2347-176x ISSN (p) 2455-0450

crossref DOI: https://dx.doi.org/10.18535/jmscr/v6i1.68

\author{
Journal Of Medical Science And Clinical Research \\ IGM Publication \\ An Official Publication of IGM Publication
}

\title{
Estimating District Specific Dengue burden in Kerala using Disability Adjusted Life Years (DALY)
}

\author{
Authors \\ Zinia T. Nujum ${ }^{1}$, Vijayakumar K. ${ }^{2}$, Meenakshy V. ${ }^{3}$, Saboora Beegum M. \\ ${ }^{1}$ Associate Professor, Community, Medicine, Government Medical College, Thiruvananthapuram \\ Present address: Associate Professor, Community, Medicine, Government Medical College, Kollam \\ ${ }^{2}$ Professor and Head, Community Medicine, Amrita Institute of Medical Sciences, Kochi \\ ${ }^{3}$ Deputy Director, National Vector borne Disease Control Programme, Directorate of Health Services, \\ Kerala \\ ${ }^{4}$ Professor and Head, Biochemistry, Government Medical College, Thiruvananthapuram
}

\begin{abstract}
Introduction: Kerala is hyperendemic for dengue. Determining State and district specific DALY are crucial in prioritizing, implementing and evaluating actions, but are seldom done. The objective of this study was to compute and compare the DALY due to Dengue in the districts of Kerala for 2014 and 2015.

Methods: DALY was computed using the DALY package, accessible in the R statistical program. The parameters that were inputed are incidence/1000, mortality rate, mean age of mortality, disability weights, proportion of cases treated. Reported incidence/1000 and mortality rates were calculated using the reported cases and deaths from the state surveillance unit. Estimates of dengue incidence/1000 were made using the fever surveillance and sentinel surveillance data. DALY was computed based on reported and estimated dengue burden. Absolute DALY $(A D)$ and Relative DALY (RD) with their $95 \%$ CI were computed.

Results: $A D$ and $R D$ was high for some districts and low for others. Thrissur, had the highest in 2014 (AD- 840, RD-26.91/lakh population [95\%CI: 11.50-39.60]) and Kasargode (AD-2601; RD-198.95/lakh [95\%CI: 85.67291.42]) in 2015.Thiruvananthapuram recorded the second highest DALY consistently for both years (AD- 801; RD-24.26/lakh [95\% CI: 11.27-35.04] and AD-2312; RD-70.03[95\% CI: 30.68-102.71]). Districts with highest incidence were not the same as those with highest DALY.

Conclusion: There is gross inequity in the burden of Dengue in the districts of Kerala. Estimated DALY in some districts are very much higher than the global estimates. Prioritization for action can be misleading if incidence is used as measure of burden.

Keywords: Dengue, DALY, Kerala, Districts, Burden of disease.
\end{abstract}

\section{Introduction}

Dengue is one of the most serious and fast emerging tropical mosquito borne disease. In Kerala cases of dengue with some deaths were reported in 1997 for the first time. The First epidemic occurred in 2003 with 3546 cases and 68 deaths. Thiruvananthapuram district was worst affected in this epidemic. In 2003, Kerala reported maximum deaths due to dengue in India. Over the years, the reported cases of dengue have been increasing. ${ }^{4}$ Kerala is now hyperendemic for Dengue with presence of multiple serotype, high rates of coinfection and local genomic evolution of viral strains. ${ }^{5}$ The district of Thiruvananthapuram reports maximum number of cases in the state. ${ }^{4}$ 
In 1992, the World Health Organization (WHO), the World Bank, and Harvard School of Public Health cooperated to develop a new metric of disability-adjusted life years (DALY) to quantify the burden of disease and injury on human beings for the Global Burden of Disease Project (GBDP). ${ }^{5,6}$ It incorporates both disability and mortality. DALY enables comparison of disease burden across regions. We can also compare the disease burden due to different health conditions using DALY. Dengue related DALY has been calculated globally. However it has not been ascertained for the country and the state. Indian Council of Medical Research ${ }^{7}$ has brought in an initiative to determine state specific DALY, but dengue specific DALY has not has not been specified. This study is an attempt to calculate the district specific dengue burden using DALY in Kerala.

\section{Methods}

DALY was computed using The DALY package, accessible in the $\mathrm{R}$ statistical program. ${ }^{8} \mathrm{~A}$ Graphical User Interface (GUI) ${ }^{9}$ is available for calculating DALYs and performing uncertainty and sensitivity analyses.

The age sex distribution of Kerala ${ }^{10}$ population was used. Life expectancy(LE) for Kerala (20112015 ) gives values only upto $85+, \mathrm{LE}$ at 90 and 95 years were retained as in LE table of $\mathrm{R}$ and taken as 2.7 and 1.9 corrected to one digit.

The reported cases of Dengue and deaths for the years 2014 and 2015 for the districts of Kerala was obtained from the state surveillance unit. The line list of cases with age and sex specific details were also obtained. Instead of using multiplication factor, we used the data from sentinel surveillance and fever surveillance to compute the highest (20\% of fever cases; may be applicable to Thiruvananthapuram only during epidemic period) and lowest possible $(2.8 \%$ of fever; applicable to the whole state) dengue estimates. These estimates of Dengue in Kerala have been published earlier by the same authors. ${ }^{11}$ The incidence/ 1000 and mortality /1000 population was calculated for the and the imputed in $\mathrm{R}$ for DALY calculation. The age sex specific incidence /1000 was calculated. The age sex profile of dengue cases, mean age of onset in each age group and mean age of mortality were computed in SPSS version 11. This data was used for DALY calculation for the corresponding years. We assumed that deaths due to Dengue may not be missed to reporting. Disability weights (DW) of GBD 1990 was used, since it has age specific values.

Since severity of disease was not given in the line list of cases reported, it was accounted in the duration of disease and disability weights. From a 5 year evaluation of dengue cases, it was seen that $90 \%$ of cases are not severe and only $10 \%$ is severe. ${ }^{12}$ The duration disease for classic dengue is 4 days (range; 2-6), moderate dengue is 10 days (range; 8-12) and 14 days for severe dengue (range; $10-18$ days). ${ }^{13}$ We took duration of disease as 7 days, based on a weighted proportion of disease severity. Others have used a duration of 5 days $^{14}, 14$ days for hospitalized patients and 4.5 days for ambulatory cases. ${ }^{13}$ Proportion of treated cases was taken as $90 \%$, because in Kerala treatment seeking is high. After finding the absolute DALY, relative DALY /lakh population was computed for comparison. We categorised districts as high, intermediate and low risk based on incidence, mortality and DALY.

This study was part of a larger study funded by WHO-TDR. Ethics committee clearance was obtained from WHO (protocol ID B40138 last approved on 27/4/2016) and institution (Govt. Medical College, Thiruvananthapuram- IEC No: 02/42/MCT dated 14/02/2014).

\section{Results}

The risk of dengue incidence, mortality and DALY across districts were different. Those districts with highest incidence were not the same as those with highest DALY. The incidence of dengue based on reported cases varied from 0.014 to $0.388 / 1000$. In 2014 incidence of dengue was highest in Thiruvananthapuram district (TVPM) 


\section{JMSCR VoI||06||Issue||01||Page 31814-31821||January}

and lowest in Palakkad (table 1).Mortality rate/ 1000 varied from zero to 0.0015 . Mean age of mortality was 38 years. In 2014, mortality was highest in Thrissur and lowest in Pathanamthitta. Thrissur had the highest Relative DALY 26.91(11.50-39.60), although TVPM had the highest incidence. This is because mortality was higher in Thrissur than TVPM. The lowest DALY was for Kottayam (Table 2).

In 2015 the highest incidence was in Kasargode followed by Thiruvananthapuram. (Table 3). The values ranged from 0.05 in Kottayam to 0.364 in Kasargode. The highest mortality was also in Kasargode (0.003/1000). Mean age of mortality was 34.87. Absolute DALY was highest in Kasargode (2601), but the relative DALY was highest for Idukki (204.3), which had only $6^{\text {th }}$ rank in terms of incidence and $3^{\text {rd }}$ rank in terms of mortality, because the population of Idukki is less.
The relative DALY of three districts in 2015 were very high. (Idukki 204.33(86.66-301.27); Kasargode -RD-198.95/lakh [95\%CI:85.67291.42]; Thiruvananthapuram RD- 70.03[95\% CI: 30.68-102.71]). At the same time there were districts with RD as low as 0.10 and 0.24 /lakh. (Table 4).

Estimated burden is much higher than burden based on reported cases during both years (Figures 1 and 2)

The number of districts with high risk incidence, mortality and DALY increased in 2015 compared to 2014. In 2014 there were only two districts with relative DALY > 20, whereas in 2015 there were 6 districts. Incidence/1000 of $>0.05$ was seen only in four districts in 2014 whereas in 2015 all except two districts had high incidence. Similarly mortality/1000 was $>0.001$ in two districts only in 2014, which increased to 6 and mortality of (Figure 3).

Table 1: Dengue cases and Incidence in the districts in 2014 compared

\begin{tabular}{|c|c|c|c|c|c|c|c|c|c|c|}
\hline$\frac{0}{z}$ & 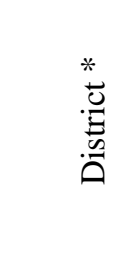 & 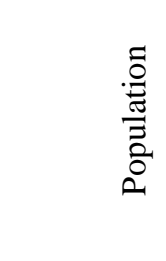 & 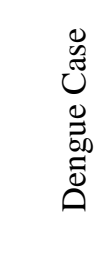 & 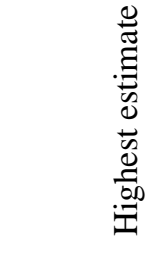 & 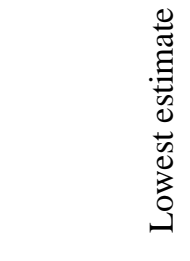 & 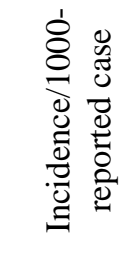 & 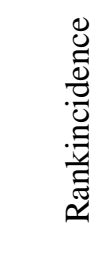 & 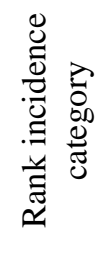 & 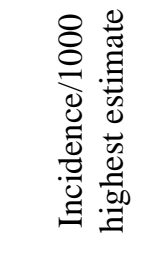 & 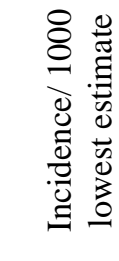 \\
\hline 1 & TVPM & 3301427 & 1280 & 59719 & 8360.66 & 0.388 & 1 & & 18.089 & 2.532 \\
\hline 2 & KLM & 2635375 & 73 & 41869 & 5861.66 & 0.028 & 9 & & 15.887 & 2.224 \\
\hline 3 & PTN & 1197412 & 191 & 18108 & 2535.12 & 0.160 & 2 & & 15.123 & 2.117 \\
\hline 4 & ALP & 2127789 & 46 & 29785.6 & 4169.984 & 0.022 & 11 & & 13.998 & 1.960 \\
\hline 5 & KTM & 1974551 & 30 & 18072.6 & 2530.164 & 0.015 & 13 & & 9.153 & 1.281 \\
\hline 6 & IDK & 1108974 & 20 & 17396.6 & 2435.524 & 0.018 & 12 & & 15.687 & 2.196 \\
\hline 7 & ERN & 3282388 & 149 & 41512.6 & 5811.764 & 0.045 & 6 & & 12.647 & 1.771 \\
\hline 8 & TSR & 3121200 & 153 & 56572.2 & 7920.108 & 0.049 & 5 & & 18.125 & 2.538 \\
\hline 9 & PKD & 2809934 & 40 & 49926.4 & 6989.696 & 0.014 & 14 & & 17.768 & 2.487 \\
\hline 10 & MLP & 4112920 & 146 & 74823.2 & 10475.248 & 0.035 & 7 & & 18.192 & 2.547 \\
\hline 11 & KZD & 3086293 & 276 & 42306.6 & 5922.924 & 0.089 & 3 & & 13.708 & 1.919 \\
\hline 12 & WYD & 817420 & 44 & 27314.4 & 3824.016 & 0.054 & 4 & & 33.415 & 4.678 \\
\hline 13 & KNR & 2523003 & 55 & 46575.6 & 6520.584 & 0.022 & 10 & & 18.460 & 2.584 \\
\hline 14 & KSD & 1307375 & 45 & 24311.4 & 3403.596 & 0.034 & 8 & & 18.596 & 2.603 \\
\hline
\end{tabular}

*TVPM - Thiruvananthapuram, KLM - Kollam, PTN- Pathanamthitta, ALP- Alappuzha, KTM- Kottayam , IDK - Idukki, ERNErnakulam, TSR- Thrissur, PKD - Palakkad, MLP- Malappuram, KZD- Kozhikkode, WYD - Wyanad, KNR- Kannour, KSDKasargode. 
Table 2: Dengue deaths, mortality rate and DALYs in the districts in 2014 compared

\begin{tabular}{|c|c|c|c|c|c|c|c|c|}
\hline 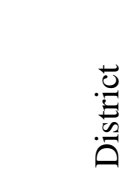 &  & 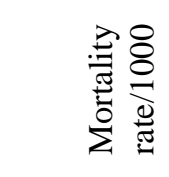 & 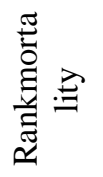 & 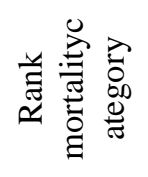 & 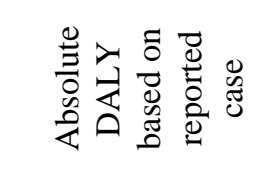 & 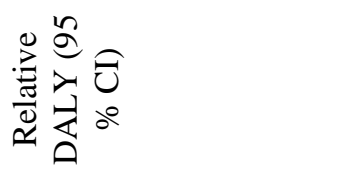 & 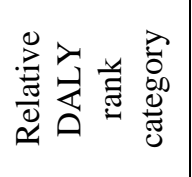 & 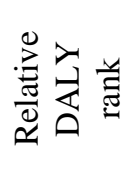 \\
\hline TVPM & 3 & 0.000909 & 3 & & $801(372-1157)$ & $24.26(11.27-35.04)$ & & 2 \\
\hline KLM & 1 & 0.000379 & 5 & & $252(106-371)$ & $9.56(4.02-14.07)$ & & 6 \\
\hline PTN & 0 & 0 & 14 & & 21 & $1.75(1.75-1.75)$ & & 9 \\
\hline ALP & 1 & 0.00047 & 4 & & $336(147-494)$ & $15.79(6.91-23.21)$ & & 3 \\
\hline KTM & 0 & 0 & 13 & & 2 & $0.10(0.10-0.10)$ & & 14 \\
\hline IDK & 0 & 0 & 12 & & 158 & $14.25(14.25-14.25)$ & & 4 \\
\hline ERN & 0 & 0 & 11 & & 6 & $0.18(0.18-0.18)$ & & 12 \\
\hline TSR & 4 & 0.001282 & 2 & & $840(359-1236)$ & $26.91(11.50-39.60)$ & & 1 \\
\hline PKD & 1 & 0.000356 & 6 & & $251(108-371)$ & $8.93(3.84-13.20)$ & & 7 \\
\hline MLP & 1 & 0.000243 & 7 & & $171(75-250)$ & $4.16(1.82-6.07)$ & & 8 \\
\hline KZD & 0 & 0 & 10 & & 12 & $0.39(0.39-0.39)$ & & 11 \\
\hline WYD & 0 & 0 & 9 & & 7 & $0.86(0.86-0.86)$ & & 10 \\
\hline KNR & 0 & 0 & 8 & & 3 & $0.11(0.11-0.11)$ & & 13 \\
\hline KSD & 2 & 0.00153 & 1 & & 132(59-193) & $10.10(4.51-14.76)$ & & 5 \\
\hline
\end{tabular}

Table 3: Dengue cases and Incidence in the districts in 2015 compared

\begin{tabular}{|c|c|c|c|c|c|c|c|c|c|c|}
\hline$\frac{i}{\dot{z}}$ & 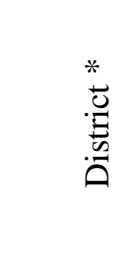 & 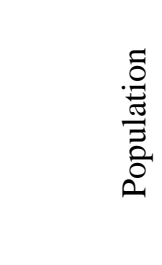 & 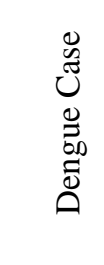 & 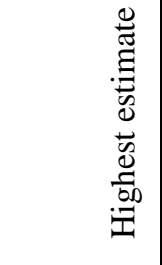 & 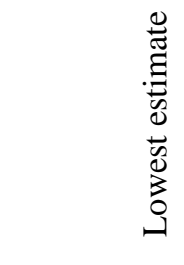 & 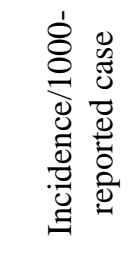 & 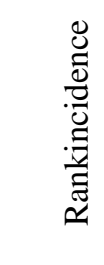 & 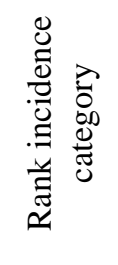 & 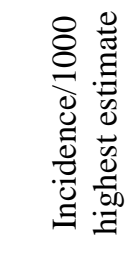 & 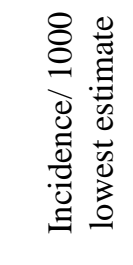 \\
\hline 1 & TVPM & 3301427 & 991 & 64862.4 & 9080.736 & 0.300 & 2 & & 19.647 & 2.751 \\
\hline 2 & KLM & 2635375 & 245 & 45068.8 & 6309.632 & 0.093 & 7 & & 17.101 & 2.394 \\
\hline 3 & PTN & 1197412 & 204 & 16174 & 2264.36 & 0.170 & 5 & & 13.507 & 1.891 \\
\hline 4 & IDK & 2127789 & 127 & 17827.4 & 2495.836 & 0.115 & 6 & & 16.076 & 2.251 \\
\hline 5 & KTM & 1974551 & 98 & 17232.2 & 2412.508 & 0.050 & 14 & & 8.727 & 1.222 \\
\hline 6 & ALP & 1108974 & 157 & 26506 & 3710.84 & 0.074 & 10 & & 12.457 & 1.744 \\
\hline 7 & ERN & 3282388 & 243 & 35762.6 & 5006.764 & 0.074 & 9 & & 10.895 & 1.525 \\
\hline 8 & TSR & 3121200 & 253 & 54461 & 7624.54 & 0.081 & 8 & & 17.449 & 2.443 \\
\hline 9 & PKD & 2809934 & 145 & 51321.2 & 7184.968 & 0.052 & 13 & & 18.264 & 2.557 \\
\hline 10 & MLP & 4112920 & 275 & 72704.4 & 10178.616 & 0.067 & 11 & & 17.677 & 2.475 \\
\hline 11 & KZD & 3086293 & 587 & 50608.4 & 7085.176 & 0.190 & 4 & & 16.398 & 2.296 \\
\hline 12 & WYD & 817420 & 157 & 31104.2 & 4354.588 & 0.192 & 3 & & 38.052 & 5.327 \\
\hline 13 & KNR & 2523003 & 156 & 45636 & 6389.04 & 0.062 & 12 & & 18.088 & 2.532 \\
\hline 14 & KSD & 1307375 & 476 & 25337.6 & 3547.264 & 0.364 & 1 & & 19.381 & 2.713 \\
\hline
\end{tabular}




\section{JMSCR Vol||06||Issue||01||Page 31814-31821||January}

Table 4: Dengue deaths, mortality rate and DALYs in the districts in 2015 compared

\begin{tabular}{|c|c|c|c|c|c|c|c|c|}
\hline  & 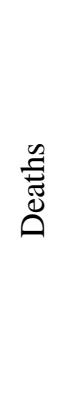 & 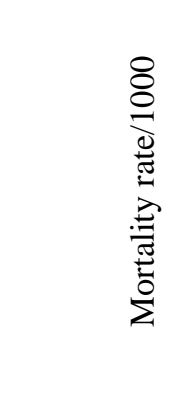 & 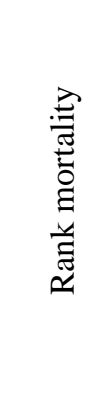 & 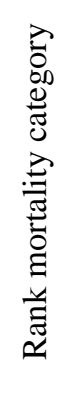 & 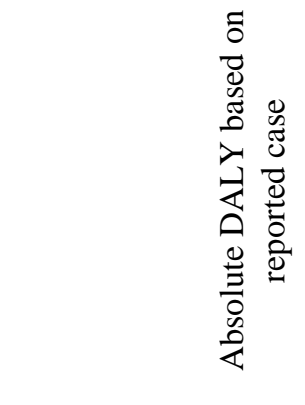 & 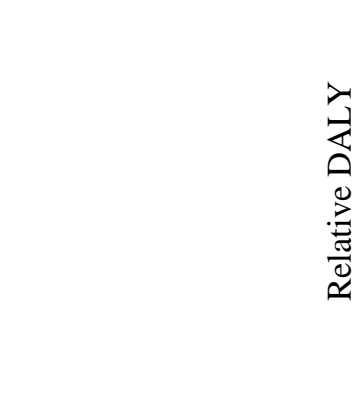 & 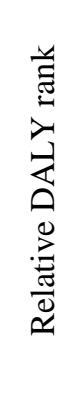 & 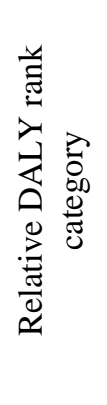 \\
\hline TVPM & 9 & 0.002726 & 2 & & $2312(1013-3391)$ & $70.03(30.06-102.71)$ & 3 & \\
\hline KLM & 3 & 0.001138 & 5 & & 958(406-1411) & $36.35(15.41-53.54)$ & 6 & \\
\hline PTN & 0 & 0 & 14 & & 23 & 1.92 & 10 & \\
\hline IDK & 3 & 0.002705 & 3 & & 2266(961-3341) & 204.33(86.66-301.27) & 1 & \\
\hline KTM & 2 & 0.001013 & 6 & & $844(357-1245)$ & $42.74(18.08-63.05)$ & 5 & \\
\hline ALP & 1 & 0.00047 & 8 & & $400(176-588)$ & $18.80(8.27-27.63)$ & 7 & \\
\hline ERN & 0 & 0 & 13 & & 10 & 0.30 & 2 & \\
\hline TSR & 0 & 0 & 12 & & 11 & 0.35 & 11 & \\
\hline PKD & 0 & 0 & 11 & & 7 & 0.25 & 14 & \\
\hline MLP & 2 & 0.000486 & 7 & & 413(181-605) & $10.04(4.40-14.71)$ & 8 & \\
\hline KZD & 5 & 0.00162 & 4 & & 1376(597-2019) & $44.58(10.34-65.42)$ & 4 & \\
\hline WYD & 0 & 0 & 10 & & 26 & 3.18 & 9 & \\
\hline KNR & 0 & 0 & 9 & & 8 & 0.32 & 12 & \\
\hline KSD & 4 & 0.00306 & 1 & & $2601(1120-3810)$ & 198.95(85.67-91.42) & 13 & \\
\hline
\end{tabular}

Figure 1: Dengue DALY based on reported cases compared to estimated burden in 2014 600

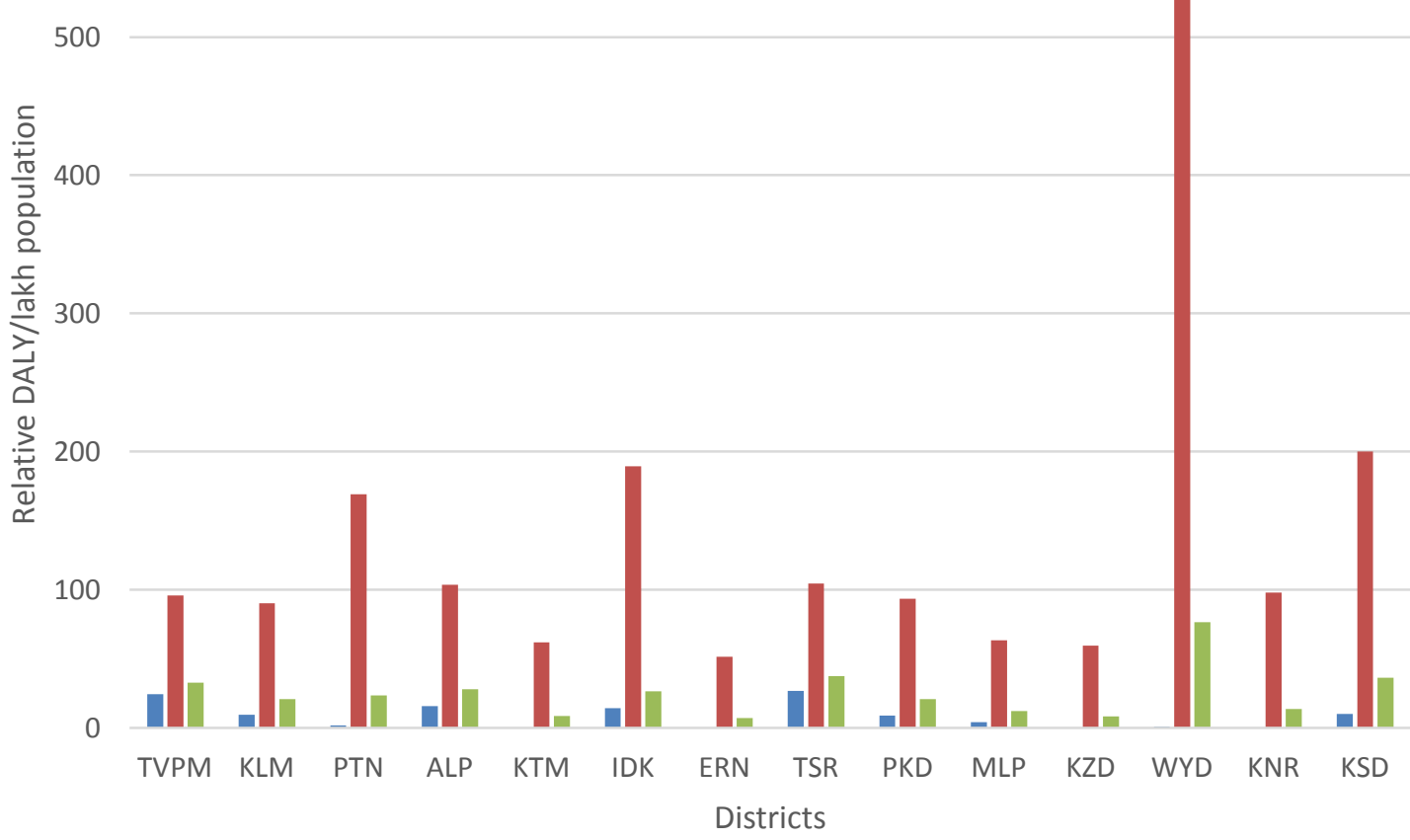

$\square$ RDRC $\square$ RDHB $\square$ RDLB 
Figure 2: Dengue DALY based on reported cases compared to estimated burden in 2015

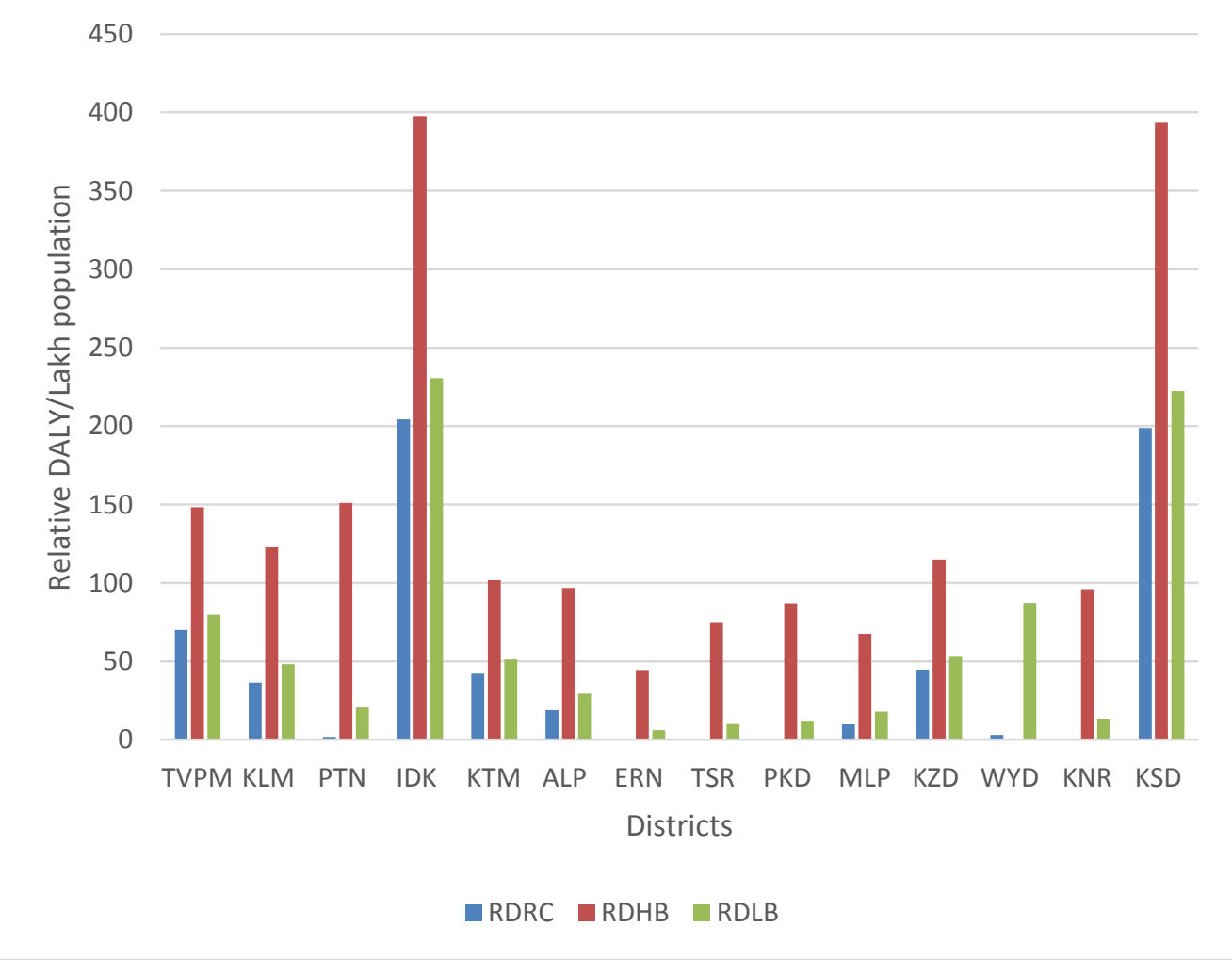

Figure 3: District wise dengue burden for the years 2014 and 2015


A. District wise Dengue related DALY in 2014
B. District wise Dengue related DALY in 2015
C. District wise Dengue Incidence in 2014
D. District wise Dengue Incidence in 2015
E. District wise Dengue mortality in 2014
F. District wise Dengue mortality 2015 


\section{Discussion}

The relative DALY according to Global burden of disease due to dengue in 2013 was $15.8 /$ lakh. $^{15}$ It increased to $25.5 /$ lakh in $2015 .{ }^{16}$ The same trend is seen in our study. The DALY of some districts in 2014 is comparable to the global burden. In 2015, however we find the DALY has escalated upto more than 8 times the global burden in two districts. The South East Asian burden is 19.5/lakh. The state DALY has been computed by us and reported in a separate publication as 7.22/lakh (6.62-7.72). Some districts have very low dengue burden. Thus there is a gross heterogeneity in the distribution of dengue burden across the state

Dengue burden is higher in all district in 2015 compared to 2014, except Thrissur and Palakkad. Although this is not a sufficient period to determine trend the increase is at par with the increase in dengue seen throughout India. Over the period 1998-2009, 82327 dengue cases (incidence: 6.34 per million population) were reported. During a more recent period (20102014), 213607 cases (incidence: 34.81 per million population) of dengue fever were observed. Thus, the number of dengue cases during the past 5 years has increased markedly, by a factor of $\sim 2.6$, with respect to the 1998-2009 period. ${ }^{17}$

Use of incidence alone may be misleading for prioritising among the districts for action. If we were to use incidence alone, the highest priority would be given to Thiruvananthapuram. However DALY estimation, which considers mortality, age at mortality, population and many other indicators besides incidence would place Thrissur district to receive the highest priority in 2014.

\section{Limitations and Strengths}

This study is the first of its kind to determine district specific dengue burden using DALY. It has relied on the state surveillance system for data on reported cases of dengue and estimation of dengue burden, besides our own personal studies. Therefore any inaccuracies in the disease reporting may also be reflected in this calculation.
Since we have calculated DALY based on reported cases and estimated cases also, any underreporting would have been taken care of.

\section{Conclusions}

There is gross heterogeneity across the districts of Kerala in dengue burden. Some districts have much higher burden than the global burden. Prioritising for action can be misleading if we use the incidence alone for burden estimation. Similarly dengue and other disease burden needs to be estimated at the district level, which can be considered as the smallest unit of planning. Strengthening surveillance system will add to the reliability of such burden estimations. We recommend the use of DALY for better understanding of distribution of disease and policy prioritization at district level.

\section{Acknowledgements}

This work was supported by World Health Organisation- Tropical Disease Research, postgraduate training grants in implementation research - 2014; TIMS ID:B40138. We place on record our gratitude to Dr. Edward Kamau for his constant guidance. He has been our contact person with WHO-TDR. Dr. Thomas Mathew, Principal, Government Medical College, Thiruvananthapuram was a pillar of support. We thank him from the depths of our heart.

\section{References}

1. Gubler, D.J. and Kuno, G. Dengue and Dengue Haemorrhagic Fever. CABI Publishing, New York, p.1,1978

2. Lall, R. and Dhanda, V. Dengue haemorrahagic fever and the dengue shock syndrome in India. Natl Med J India 9: 20, 1996.

3. Kadar A., Kandasamy, M.S., Appavoo, P. and Anuradha, C.N. Outbreak and control of dengue in a village of Dharmapuri, Tamil Nadu. J Commun Dis 29: 69, 1997.

4. State Bulletin, Thiruvananthapuram. Integrated Disease Surveillance Project, 
State Surveillance Unit, Directorate of Health Services, Government of Kerala, 2010.

5. Murray CJL. Rethinking DALYs. In: Murray CJL, Lopez AD, editors. The Global Burden of Disease. Vol 1 of Global Burden of Disease and Injury Series. Cambridge, MA: Harvard School of Public Health; 1996. pp. 1-98.

6. The Global Burden of Disease: 2004 Update. WHO. [Accessed 2009 Feb 4]. Available

from:http://www.who.int/healthinfo/global _burden_disease/2004_report_update/en/in dex.html

7. Indian Council of Medical Research, Public Health Foundation of India and Institute for Health Metrics and Evaluation. India: Health of the Nation's States- The India State-Level Disease Burden Initiative. New Delhi:ICMR, PHFI, and IHME; 2017.

8. R Core Team (2016). R: A language and environment for statistical computing. $\mathrm{R}$ Foundation for Statistical Computing, Vienna, Austria. URL https://www.Rproject.org/.

9. Brecht Devleesschauwer, Scott McDonald, Juanita Haagsma, Nicolas Praet, Arie Havelaar and Niko Speybroeck (2016). DALY: The DALY Calculator - Graphical User Interface for Probabilistic DALY calculation in $\mathrm{R}$. $\mathrm{R}$ package version 1.5.0. https://cran.r-project.org/package=DALY

10. Office of the Registrar General and Census Commisioner, India. Ministry of Home Affairs, Government of India, Census Digital Library and Sample Registration System Publications. Available at http://censusindia.gov.in/2011-

common/censusdataonline.html. Accessed on 15-06-2017.

11. Zinia $\mathrm{T}$ Nujum, Achu Thomas, Vijayakumar K, Thomas Mathew, Saran S
Pillai. Estimation of Burden Using Active Dengue Sentinel Surveillance and Fever Surveillance. Dengue Bulletin Volume 39December 2016; pages 78-68.

12. Cecilia D. Current status of dengue and chikungunya in India. WHO South-East Asia J Public Health 2014; 3(1): 22-27.

13. Shepard DS, Undurraga EA, Halasa YA (2013) Economic and Disease Burden of Dengue in Southeast Asia. PLoSNegl Trop Dis 7(2): e2055. https://doi.org/10.1371/journal.pntd.00020 55.

14. Beauté and Vong: Cost and disease burden of Dengue in Cambodia. BMC Public Health 2010 10:521.

15. Jeffrey D Stanaway, Donald S Shepard, Eduardo A Undurraga, Yara A Halasa, Luc E Coffeng, Oliver J Brady et al. The global burden of dengue: an analysis from the Global Burden of Disease Study 2013. Available at www.thelancet.com/infection Vol 16 June 2016, accessed on 7-07-2017.

16. GBD 2015 DALYs and HALE Collaborators. Global, regional, and national disability-adjusted life-years (DALYs) for 315 diseases and injuries and healthy life expectancy (HALE), 19902015: a systematic analysis for the Global Burden of Disease Study 2015. Lancet 2016; 388: 1603-58.

17. Srinivasa Rao Mutheneni, Andrew $P$ Morse, Cyril Caminade and Suryanaryana Murty Upadhyayula. Dengue burden in India: recent trends and importance of climatic parameters. Emerging Microbes \& Infections (2017) 6, e70; doi:10.1038/emi.2017.57. Available at https://www.nature.com/articles/emi20175 7.pdf, accessed on 7/01/2018 